\title{
Towards Minimal Barcodes
}

\author{
Rocío González-Díaz ${ }^{1}$, María-José Jiménez ${ }^{1}$, and Hamid Krim² \\ 1 Applied Math Dept., School of Computer Engineering, U. Seville, Spain \\ 2 ECE Dept., NCSU, Raleigh, NC USA \\ \{rogodi,majiro\}@us.es, ahk@ncsu.edu
}

\begin{abstract}
In the setting of persistent homology computation, a useful tool is the persistence barcode representation in which pairs of birth and death times of homology classes are encoded in the form of intervals. Starting from a polyhedral complex $K$ (an object subdivided into cells which are polytopes) and an initial order of the set of vertices, we are concerned with the general problem of searching for filters (an order of the rest of the cells) that provide a minimal barcode representation in the sense of having minimal number of " $k$-significant" intervals, which correspond to homology classes with life-times longer than a fixed number $k$. As a first step, in this paper we provide an algorithm for computing such a filter for $k=1$ on the Hasse diagram of the poset of faces of $K$.
\end{abstract}

Keywords: Persistent homology, persistence barcodes, graphs, polyhedral complexes.

\section{Introduction}

The persistence barcode representation, which encodes pairs of cells meaning birth and death of homology classes in persistent homology computation, depends on the filter considered for such computation. Although, as we will see later, the total number of intervals remains invariant, the lengths of these intervals depend on the selected filter. Since, non-significant intervals (i.e. intervals with short length) do not imply relevant homological information, we are interested in providing good properties to be satisfied by the selected filter, so that the number of non-significant intervals in the corresponding persistence barcode is maximized (i.e. the number of significant intervals is minimized). Motivated by practical applications of persistent homology computation, our starting point is a given polyhedral complex and an initial order of the set of vertices. From an information-theoretic viewpoint, and if we interpret the number of "significant intervals" as the coding length of a complex, our goal is to then select the most "parsimonious" representation (also by Occam's razor principle). As is also well known, the coding length is also intimately related to the notion of entropy (i.e. a topological entropy of a complex in our case). While ideally, one would want to balance this minimization with a penalty term of the number of "insignificant"

\footnotetext{
* Partially supported under grant MTM2012-32706. Authors listed alphabetically.
} 
intervals. This development is under way, and requires the statistical distribution of the long intervals. Our aim is to look for a way to insert the rest of the cells along the filter in order to minimize the number of significant intervals.

The remainder of this paper is organized as follows. Section 2 covers the relevant background material. In Section 3 , we prove that the number of intervals in a persistence barcode does not depend on the selected filter, we give the definition of minimal barcode and show two practical examples in which computation of minimal barcodes can be useful. In Section 4, an algorithm for computing on the Hasse diagram of the poset of faces of the polyhedral complex, a filter that produces a minimal barcode is given. Section 5 is devoted to relations between minimal barcodes and the optimal discrete Morse function. Conclusions and future work are presented in Section 6 .

\section{Background}

This paper is developed in a combinatorial algebraic topology setting, where the objects of interest are (geometric) polyhedral complexes [14. A polyhedral complex $K$ is a collection of convex polytopes such that: (1) every face of a polytope in $K$ is itself a polytope in $K$; (2) the intersection of any two polytopes in $K$ is a face of each of them. The set of cells of dim. $i$ (or $i$-cells), $\sigma^{i}$ (superscript specifies its dimension), will be denoted by $K^{i}$, and the number of cells in the set of cells $S$ by $|S|$. An $n$-dim. polyhedral complex satisfies that $K^{n} \neq \emptyset$ and $K^{n+1}=\emptyset$, where $n>0$. Particular cases arise when the polytopes belong to a specified set of polyhedra, such as simplicial complexes (vertices, edges, triangles, tetrahedra, up to dim. 3) or cubical complexes (vertices, edges, squares, cubes, up to dim. $3)$. In general, we will always refer to a finite polyhedral complex $K$ (i.e. with $m$ cells, $m$ being a finite number). Several polytopes associated with combinatorial optimization problems have surprisingly small extended formulations (see [3 20 10] . It may not be very surprising that no polynomial size extended formulations of polytopes associated with NP-hard optimization problems like the traveling salesman polytope are known.

Homology theory uses algebraic groups to encode the topological structure of $K$. Finite formal sums of elements of $K^{i}$ (called $i$-chains) define an additive abelian group structure on $K^{i}$. A proper face of $\sigma \in K^{i}$ is a face of $\sigma$ of dim. $i-1$. The boundary of $\sigma$, denoted by $\partial(\sigma)$ is the formal sum (with coefficients in $\mathbb{Z} / \mathbb{Z} 2$ ) of the proper faces of $\sigma$. The boundary operator is extended to all chains of $K$ by linearity. An $i$-chain $a$ is an $i$-cycle if $\partial(a)=0$; it is an $i$-boundary if there is an $(i+1)$-chain $b$ such that $\partial(b)=a$. Two $i$-cycles $a$ and $a^{\prime}$ are homologous if $a+a^{\prime}$ is an $i$-boundary. The quotient of $i$-cycles over $i$-boundaries is the $i^{t h}$ homology group of $K$. The $i$-Betti number that is the rank of the $i^{\text {th }}$ homology group of $K$ will be denoted by $\beta^{i}$. Then, the basic topological structure of $K$ is quantified by the number of independent cycles in each homology group. See [17 12.

Persistent homology [4|21] studies homology classes and their lifetimes (persistence). While homology characterizes an object, persistent homology characterizes a sequence of growing object-instances, i.e. an object together with an 

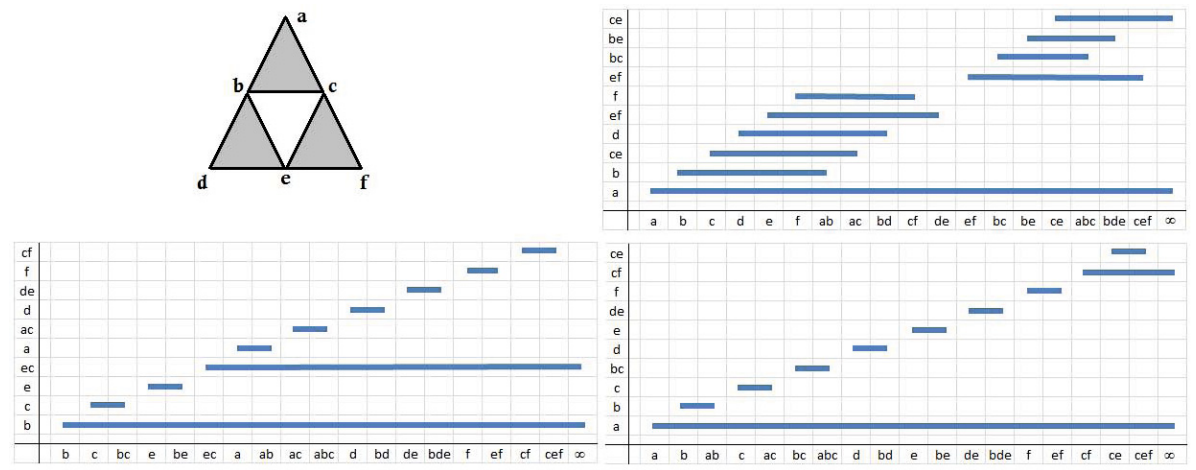

Fig. 1. An example of a 2-dim. simplicial complex and three persistence barcodes corresponding to different filters (fixed by the order in the set of cells given along the horizontal axis of the representations). Bottom: two minimal barcodes. Right: two barcodes with same order of the 0-cells.

order of the cells $\left\{\sigma_{1}, \ldots, \sigma_{m}\right\}$ (where $\sigma_{s}<\sigma_{t}$ iff $s<t$ ) called a filter, such that if $\sigma_{i}$ is a proper faces of $\sigma_{j}$ then $i<j$.

Given a filter of $K$, the algorithm for computing persistent homology that appears in 4], marks an $i$-cell $\sigma_{t}$ as positive (birth) if it belongs to an $i$-cycle in $K_{t}\left(\sigma_{t}\right.$ creates a new homology class at time $\left.t\right)$ and negative (death) otherwise ( $\sigma_{t}$ destroys the homology class created at some time $s$ for $0 \leq s<t$ ).

Given a filter $\left\{\sigma_{1}, \ldots, \sigma_{m}\right\}$, a persistence barcode [2] is a graphical representation of pairs of birth and death times as a collection of horizontal line segments (intervals) in a plane. If a cell $\sigma_{s}$ creates a homology class at time $s$, and it is destroyed at time $t, 0 \leq s<t \leq m$ then the interval $[s, t)$ is added to the corresponding persistence barcode (see [2]); If a cell $\sigma_{s}, 0 \leq s \leq m$ creates a homology class at time $s$ and it survives along the process, then the interval $[s, \infty)$ is added to the persistence barcode.

\section{Minimal Barcodes}

In this section, a formal definition of minimal barcode is presented along with two practical examples for which a computation of minimal barcodes can be useful.

For a fixed $i$, we refer to $i$-barcode the set of intervals of a given persistence barcode corresponding to the pairs of positive $i$-cells and negative $(i+1)$-cells of $K$. The following result holds.

Lemma 1. The number of intervals in an $i$-barcode, $0 \leq i \leq n$, is constant, independently of the selected filter.

Proof. First, the number of intervals of infinite length in the $i$-barcode is independent on the filter since it coincides with $\beta^{i}$. Second, each $i$-cell $\sigma_{t}^{i}$ in the 
given filter, $0 \leq t \leq m$, is marked as positive or negative. No cell can remain unmarked after the whole process. This is easy to prove using AT-models [6] $\sigma_{t}^{i}$ is marked as positive if $f^{i-1} \partial\left(\sigma_{t}^{i}\right)=0$ and negative otherwise (see [7]). Third, let $B^{i}$ (resp. $D^{i}$ ) be the number of positive (resp. negative) $i$-cells. Then, we have that $\left|K^{0}\right|=B^{0},\left|K^{n}\right|=B^{n}+D^{n}, \beta^{n}=B^{n}$, and, for $0<i<n,\left|K^{i}\right|=B^{i}+D^{i}$ and $\beta^{i}=B^{i}-D^{i+1}$. Therefore, we obtain that $D^{0}=0, B^{0}=\left|K^{0}\right|$, and for $0<i \leq n, D^{i}=\left|K^{i-1}\right|-\beta^{i-1}-D^{i-1}$ and $B^{i}=\left|K^{i}\right|-D^{i}$. We conclude that, for $0 \leq i \leq n$, nor $B^{i}$ neither $D^{i}$ (which coincides with the number of finite intervals in the $(i-1)$-barcode) depend on the selected filter.

A general idea in the study of topological persistence is that significant topological attributes must have long life-times, and topological features with short life-times are considered to be "noise". Following this idea, in the definition below, $k$-significant intervals correspond to homology classes whose life-times are longer than a fixed number $k$.

Definition 1. Fixed $k>0$, an interval $[s, t)$ is $k$-significant if $k<t-s$.

Our general aim is to find, under some constraints, depending on the nature of the application, filters that minimize the number of long-life homological classes which are associated with significant intervals.

Nevertheless, filters are, in many cases, totally determined. Examples of this is when objects are presented as point cloud data and Rip or Cech complexes are constructed to fill in the higher-dimensional simplices of the proximity graph whose edges are determined by proximity, i.e. vertices within some specified distance $\epsilon$ (see [8]). But, in other cases, only order of 0-cells are given. For example, when a continuous function (e.g. a height function or barycentric distance) is provided and the 0 -cells of $K$ are ordered by the function values at them.

We briefly present here two particular examples of this last case:

1. Application of persistent homology to the evaluation of a $3 \mathrm{D}$ reconstruction process (carving voxel) of human models from images captured from a set of cameras placed around the subject. In fact, we refer to the visual hull that is constructed from images of cameras from different viewpoints. This problem can be seen as a view planning problem (see [19], a survey of computer vision sensor planning, [18, a more recent survey of view planning for $3-\mathrm{D}$ vision). In our case, 9, starting from a compact block of voxels, each time a camera is added, a set of voxels are deleted (carved) from the 3D reconstruction, so the sequence of $3 \mathrm{D}$ reconstructions along decreasing number of cameras gives place to a filter of the corresponding cubical complexes. This allows to analyze the topological evolution of the reconstruction process. Only $k$ significant intervals are considered, where $k$ is the distance (in number of cells) from one reconstruction to the next one. An initial partial order is hence considered in the set of vertices that have to be added in the computation along the process. See Fig. 2 .

2. In [1] an image/video application using topological invariants for human gait recognition is shown. Using a background subtraction approach, a stack of silhouettes is extracted and glued through their gravity centers, forming 

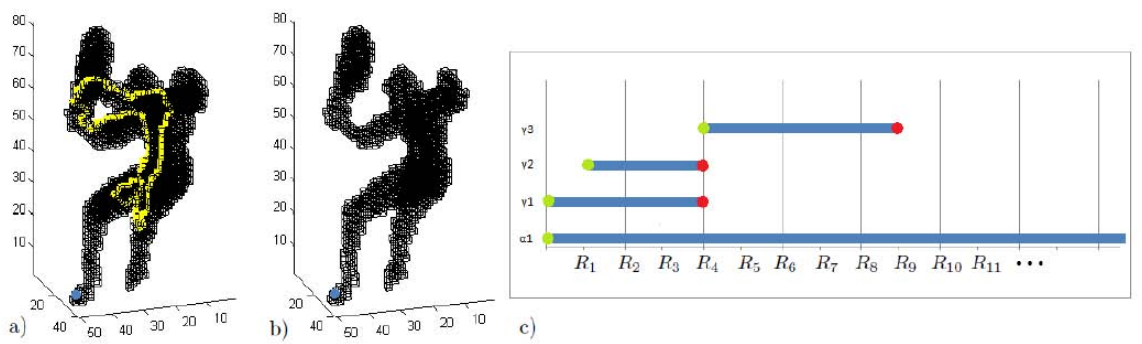

Fig. 2. Examples of 3D reconstructions using a) 4 cameras and b) 10 cameras. Representative cycles of homology are highlighted in both cases. c) Persistence barcode associated to the whole sequence of $3 \mathrm{D}$ reconstructions with increasing number of cameras (from 1 to 50) is shown.

a 3D digital image $I$. From this 3D representation, the boundary simplicial complex $\partial K(I)$ is obtained. Four filters are computed preserving four different given orders of the vertices of $\partial K(I)$ depending on four directions of view. The persistence barcodes associated with the previous filters are then computed (see Fig. 3). These filters capture relations among the parts of the human body when walking. Only intervals with long life-times are considered. Finally, a topological gait signature is extracted from the persistence barcodes according to the filters.

From now on, suppose a bijective function $h: K^{0} \rightarrow\left\{1,2, \ldots,\left|K^{0}\right|\right\}$ (i.e. an order of the 0-cells of $K$ ) is given. Let us denote by $\mathcal{F}$ the set of filters $F$ of $K$ such that for any two 0 -cells $\sigma_{s}^{0}, \sigma_{t}^{0} \in F, s<t$, it is satisfied that $h\left(\sigma_{s}^{0}\right)<h\left(\sigma_{t}^{0}\right)$.

Definition 2. A persistence barcode associated with a filter $F \in \mathcal{F}$ is minimal if the persistence barcode associated with any other filter in $\mathcal{F}$ contains greater or equal number of significant intervals.

Observe that a filter $F \in \mathcal{F}$ with a minimal barcode always exists and might not be unique (see Fig. 1 as examples of minimal barcodes).

\section{Hasse Diagrams for the Poset of Faces and Minimal Barcodes}

Our aim in this section is to construct a filter $F \in \mathcal{F}$ with a minimal barcode.

Consider the poset given by the set of cells of $K$ together with the partial order induced by the coface relation, that is, $\tau<\sigma$ if $\tau$ is a face of $\sigma$. The Hasse diagram $H$ of this poset (poset of faces) is the directed graph whose vertex set is the set of cells and whose arcs are the covering pairs $(\tau, \sigma)$ in the poset, that is, $\tau<\sigma$ and there is no $\rho$ such that $\tau<\rho<\sigma$ (it is said that $\sigma$ covers $\tau$ ). We draw the Hasse diagram in the plane in such a way that, if $\tau$ is a face of 

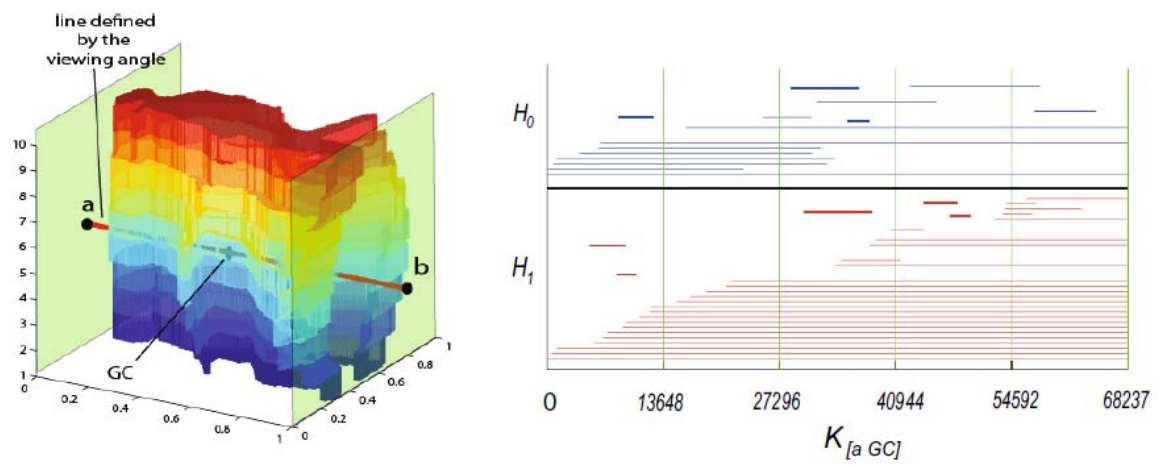

Fig. 3. Left: A simplicial complex $\partial K(I)$ corresponding to a gait, vertical direction of view (defined by the segment $[a, b]$ ) and the gravity center $G C$. Right: persistence barcode according to the vertical direction of view, corresponding to a filter of the subcomplex $K_{[a, G C]}$ (from $a$ to $G C$ ) of $\partial K(I)$.

$\sigma(\sigma$ covers $\tau)$, then the point representing $\sigma$ is in a lower level than the point representing $\tau$, corresponding the level with the dimension of the cells. Then no arrows are required in the drawing, since the directions of the arrows are implicit. $V^{i}$ denotes the set of points $v^{i}$ at level $i$, for $0 \leq i \leq n$.

In Alg. 1, a matching (or independent set of edges) $M$ in $H$ is provided, together with a vertex-labeling of $H$ (see Fig. 4). The resulting labeling and matching will produce a filter of $K$.

A weight $w^{i}\left(v^{i}\right)$ for each $v^{i} \in V^{i}, 1 \leq i \leq n$, will also be assigned along the process as follows. First, $\ell^{0}: V^{0} \rightarrow\left\{1, \ldots,\left|K^{0}\right|\right\}$ is defined for each vertex $v^{0} \in V^{0}$ by $\ell^{0}\left(v^{0}\right)=h\left(\sigma^{0}\right)$, where $\sigma^{0}$ is the 0 -cell represented by the point $v^{0}$. Second, for $i=1$ to $i=n$, the weight of each point $v^{i} \in V^{i}$ will be

$$
w^{i}\left(v^{i}\right)=\max \left\{\ell^{i-1}\left(v^{i-1}\right) \text { such that } v^{i-1} \text { is adjacent to } v^{i}\right\} .
$$

Observe that more than one point in $V^{i}$ can have the same weight. Then, a matching between vertices of $V^{i-1}$ and vertices of $V^{i}$ is given satisfying that if $v^{i-1}$ is matched with $v^{i}$ then $\ell\left(v^{i-1}\right)=w\left(v^{i}\right)$. Observe that fixing a weight $w$, only one point of the set $W=\left\{v^{i} \in V^{i}\right.$ such that $\left.w^{i}\left(v^{i}\right)=w\right\}$ is matched with some point in $V^{i-1}$. At the end of the process, a bijective function $\ell^{i}: V^{i} \rightarrow$ $\left\{1, \ldots,\left|K^{i}\right|\right\}, 1 \leq i \leq n$, is obtained, satisfying:

$\mathrm{P} 1$ if $w^{i}\left(u^{i}\right)<w^{i}\left(v^{i}\right)$, then $\ell^{i}\left(u^{i}\right)<\ell^{i}\left(v^{i}\right)$ for any $u^{i}, v^{i} \in V^{i}$;

$\mathrm{P} 2$ if $w^{i}\left(v^{i}\right)=w^{i}\left(u^{i}\right)$ and $\left(v^{i-1}, v^{i}\right) \in M$ for some $v^{i-1} \in V^{i-1}, v^{i}, u^{i} \in V^{i}$ then $\ell^{i}\left(v^{i}\right)<\ell^{i}\left(u^{i}\right)$ (any point in $V^{i}$ matched with a point in $V^{i-1}$ always precedes the other points in $V^{i}$ with same weight);

P3 if $w^{i}\left(v^{i}\right)=w^{i}\left(u^{i}\right), v^{i}, u^{i} \in V^{i}$ and $\left(v^{i}, v^{i+1}\right) \in M$ for some $v^{i+1} \in V^{i+1}$ then $\ell^{i}\left(u^{i}\right)<\ell^{i}\left(v^{i}\right)$ (points in $V^{i}$ matched with points in $V^{i+1}$ go after other points in $V^{i}$ not matched with any point in $V^{i+1}$, with same weight);

P4 Points in $V^{i}$ with same weight have consecutive labels. 

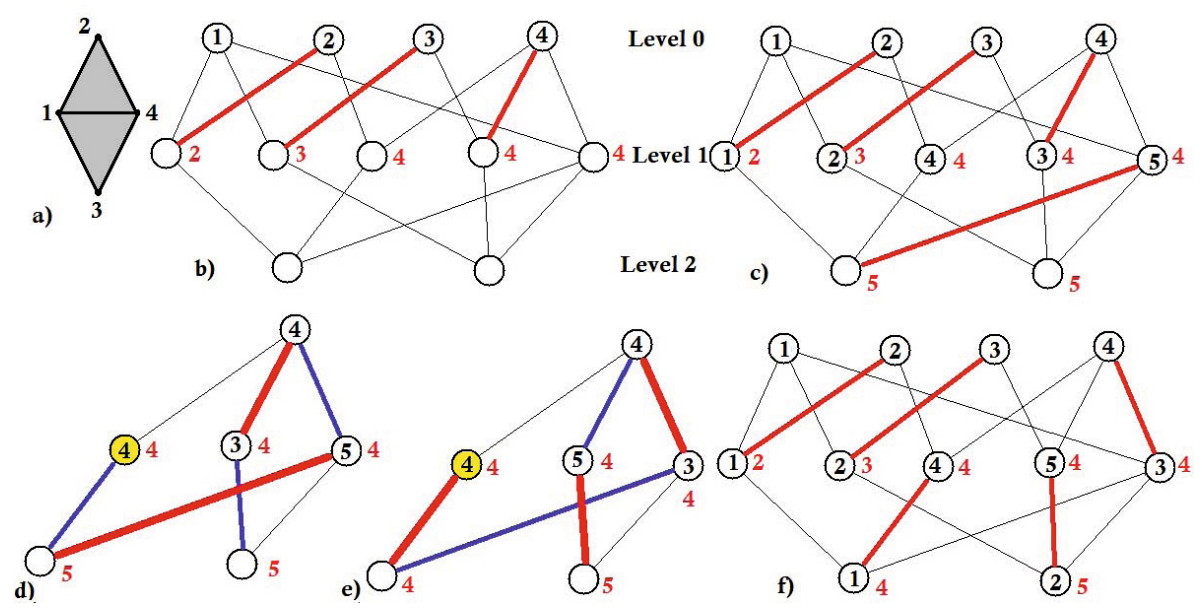

Fig. 4. a) An example of a 2-dim. simplicial complex; b) 1st step $(i=1)$ of Alg. 1 (red edges represent matchings); c) 2nd step $(i=1)$ of Alg. 11 d) subgraph $H_{v_{3}}$ and an augmenting path (blue and red edges); e) a maximal matching for $H_{v_{3}}$ (red edges); f) final matching $M$ on $H$.

P1, P2 and P3 will guarantee that the corresponding filter is correct in the sense that a cell is not added to the filter until all its faces are added.

Algorithm 1. Computing a vertex-labeling and a matching $M$ of $H$.

a. Labeling $\ell^{0}: V^{0} \rightarrow\left\{1,2, \ldots,\left|K^{0}\right|\right\}$ is given by the corresponding initial order of the 0 -cells of $K$.

b. Assign the weight $w^{1}\left(v^{1}\right)$ to each point $v^{1} \in V^{1}$.

c. $M=\left\{\left(v^{0}, v^{1}\right)\right.$ satisfying that $\left.v^{0} \in V^{0}, v^{1} \in V^{1}, \ell^{0}\left(v^{0}\right)=w^{1}\left(v^{1}\right)\right\}$. If there are more than one point in $V^{1}$ with the same weight, select one of them to match.

d. Construct $\ell^{1}: V^{1} \rightarrow\left\{1,2, \ldots,\left|K^{1}\right|\right\}$ satisfying P1, P2, P4.

Repeat the following process for $i=1$ to $i=n-1$ :

1. Assign the weight $w^{i+1}\left(v^{i+1}\right)$ to each point $v^{i+1} \in V^{i+1}$.

2. Update matching:

$M:=M \cup\left\{\left(v^{i}, v^{i+1}\right)\right.$ such that $\left.v^{i} \in \bar{M}^{i}, v^{i+1} \in V^{i+1}, \ell^{i}\left(v^{i}\right)=w^{i+1}\left(v^{i+1}\right)\right\}$,

where $\bar{M}^{i}$ is the set of points of $V^{i}$ not-matched with any point of $V^{i-1}$. If there are more than one point in $V^{i+1}$ with the same weight, select one of them to match.

3. For each $v^{i-1} \in V^{i-1}$, consider the subgraph $H_{v^{i-1}}$ whose set of vertices is: $A \cup B \cup C$ where $A=\left\{v^{i-1}\right\}, B=\left\{v^{i} \in V^{i}\right.$ such that $\left.w^{i}\left(v^{i}\right)=\ell^{i-1}\left(v^{i-1}\right)\right\}$ and $C=\left\{v^{i+1} \in V^{i+1}\right.$ such that $w^{i+1}\left(v^{i+1}\right)=\ell^{i}\left(v^{i}\right)$ for some point $v^{i} \in V^{i}$ satisfying that $\left.w^{i}\left(v^{i}\right)=\ell^{i-1}\left(v^{i-1}\right)\right\}$. 
3.1. If the matching $\left.M\right|_{H_{v^{i-1}}}$ (i.e., $M$ restricted to $H_{v^{i-1}}$ ) is not maximal in $H_{v^{i-1}}$, find a maximal one, $M_{v^{i-1}}$, using the augmenting path algorithm, with the restriction that each augmented path always begins in a notmatched vertex $v^{i} \in H_{v^{i-1}} \cap V^{i}$. This last restriction will guarantee that if $v^{i-1}$ was not matched in $H_{v^{i-1}}$, it remains unmatched in $H_{v^{i-1}}$.

3.2. Remove from $M$ the pairs in $\left.M\right|_{H_{v i-1}}$ and add to $M$ the pairs in $M_{v^{i-1}}$. 4. Update $\ell^{i}: V^{i} \rightarrow\left\{1,2, \ldots,\left|K^{i}\right|\right\}$ satisfying P1, P2, P3, P4.

5. For each point $v^{i+1} \in V^{i+1}$, update weight $w^{i+1}\left(v^{i+1}\right)$ if needed.

6. Construct $\ell^{i+1}: V^{i+1} \rightarrow\left\{1,2, \ldots,\left|K^{i+1}\right|\right\}$ satisfying P1, P2, P4.

Observe that a labeling $\ell^{i}$ satisfying P1, P2, P3, P4 (Step 4 in the description of the algorithm above) can always be obtained. First, before updating, labeling $\ell^{i}$ satisfied P1, P2, P4 (Step 6). Second, to satisfy P3, we just interchange the labels between points in $V^{i}$ with same weight, then the updated labeling $\ell^{i}$ will also P3 and also P1, P2 and P4.

Since the points $v^{i} \in V^{i}$ correspond to $i$-cells in $K, 0 \leq i \leq n$, an order of all the points in the planar representation of the Hasse diagram of $K$ provide a filter of $K$. Such an order $O$ can be constructed as follows:

Algorithm 2. Computing a filter $F$ of $K$ form a vertex-labeling and matching $M$ of $H$ obtained in Alg. 1.

1. Initially $O$ is the ordered set of all the points in $V^{0}$ ordered by their labels.

2. For $i=1$ to $i=n$ do:

(a) For every point $v^{i} \in V^{i}$ matched with a point $v^{i-1} \in V^{i-1}$, insert $v^{i}$ in $O$ just after $v^{i-1}$ (this way, the $i$-cell associated with the point $v^{i}$ is added to the filter $F$ just after its last face is added).

(b) Add the rest of the points in $V^{i}$ at the end of $O$ ordered by their labels.

Remark 1. The number of non-significant intervals in the filter $F$ given by the order $O$ coincides with the number of pairs in $M$.

Proposition 1. Fixing a filter $F$ of $K$ up to dimension $i-1$ (i.e., a filter of $\left.\bigcup_{j=0}^{i-1} K^{j}\right)$, Step 3 in the description of Alg. 1 produces a minimal $i$-barcode.

Proof. Observe that fixing a labeling and matching up to level $i-1$, Setp 3 of Alg. 1 produces a maximal matching between points at level $i-1, i$ and $i+1$, with the condition that points in $v^{i-1}$ already matched with points in $V^{i-2}$ are not matched with any point in $V^{i}$.

Implementation of the above algorithms is an ongoing work. Based on the previous proposition and some preliminary computations our conjecture is that the procedure explained above produces a filter with a minimal barcode.

\section{Relations between Minimal Barcodes and Optimal Discrete Morse Function}

Discrete Morse functions on cell complexes were defined by Forman in [5]. A function, $f: K \rightarrow \mathbb{R}$ is a discrete Morse function if for every $\sigma \in K, f$ takes a 
value less than or equal to $f(\sigma)$ on at most one coface of $\sigma$ and takes a value greater than or equal to $f(\sigma)$ on at most one face of $\sigma$. A cell $\sigma$ is critical if all cofaces take strictly greater values and all faces are strictly lower. A discrete vector field $V$ is a collection of pairs $\left(\sigma^{i}<\sigma^{i+1}\right)$ of cells in $K$ such that each cell is in at most one pair of $V$. A discrete Morse function defines a discrete vector field by pairing $\sigma^{i}<\sigma^{i+1}$ whenever $f\left(\sigma^{i}\right) \geq f\left(\sigma^{i+1}\right)$. The critical cells are precisely those that do not appear in any pair. Discrete vector fields that arise from Morse functions are called gradient vector fields. A $V$-path is a sequence of cells: $\sigma_{0}^{i}, \sigma_{0}^{i+1}, \sigma_{1}^{i}, \sigma_{1}^{i+1}, \ldots \sigma_{r-1}^{i}, \sigma_{r-1}^{i+1}, \sigma_{r}^{i}$ where $\left(\sigma_{t}^{i}, \sigma_{t}^{i+1}\right) \in V, \sigma_{t}^{i+1}>\sigma_{t+1}^{i}$ and $\sigma_{t}^{i} \neq \sigma_{t+1}^{i}$. A $V$-path is a non-trivial closed $V$-path if $\sigma_{r}^{i}=\sigma_{0}^{i}$ for $r \geq 1$. Forman shows that a discrete vector field is the gradient vector field of a discrete Morse function if and only if there are no non-trivial closed $V$-paths.

There have been several works in the literature dealing with the problem of obtaining optimal discrete Morse functions (the function has the minimum possible number of critical cells in each dimension) and perfect Morse function (the number of critical $i$-cells coincides with the ith Betti number of the complex). In 13 it is shown that computing optimal Morse matchings in the setting of simplicial complexes is NP-hard. In [15], a linear algorithm to define optimal discrete Morse functions on discrete 2-manifolds is introduced. In [1 the authors establish conditions under which a 2-dim. simplicial complex admits a perfect discrete Morse function and conversely.

It is clear that our work presents similarities with the problem of the computation of optimal discrete Morse functions. But in our case, a fixed ordering on the 0 -cells are given. Then, optimal discrete Morse matchings could not produce minimal barcodes (indeed, could not produce valid filters) and viceversa, the set of non-significant intervals in a minimal barcode could not be an optimal discrete Morse matching.

\section{Conclusions and Future Work}

In this paper, starting from a polyhedral complex $K$ and an initial order of the set of vertices, we provide an algorithm for computing a filter on the Hasse diagram of the poset of faces of $K$ such that the associated persistence barcode representation has a minimal number of significant intervals which correspond to homology classes with life-times longer than 1 .

An idea to adapt the presented algorithm to compute minimal barcodes to the case in which significant intervals are intervals with length greater than $k$, for $k>1$, could be: First, to compute a minimal barcode using the above algorithms. Observe that, in this case, the matched points are successively inserted in the filter whereas the non-matched points are successively added at the end. Second, modify the Hasse diagram pretending collapses of the pairs of cells associated with the non-significant intervals and apply the above algorithm again. Observe that in this step we only reorder the cells that have been added to the end. Third, repeat the process $k-1$ times. 


\section{References}

1. Ayala, R., Fernandez-Ternero, D., Vilches, J.A.: Perfect discrete Morse functions on 2-complexes. Pattern Recognition Letters 33, 1495-1500 (2012)

2. Carlsson, G., de Silva, V., Morozov, D.: Zigzag persistent homology and real-valued functions. In: Proc. 25th Annual Symposium on Computational Geometry (SoCG), pp. 247-256 (2009)

3. Conforti, M., Cornuejols, G., Zambelli, G.: Extended formulations in combinatorial optimization. 4OR: A Quarterly Journal of Operations Research 8(1), 1-48 (2010)

4. Edelsbrunner, H., Letscher, D., Zomorodian, A.: Topological persistence and simplification. In: Proc. 41st Annual Symposium on Foundations of Computer Science (FOCS 2000), pp. 454-463. IEEE Computer Society (2000)

5. Forman, R.: Morse theory for cell complexes. Advances in Mathematics 134, 90-145 (1998)

6. Gonzalez-Diaz, R., Real, P.: On the cohomology of 3D digital images. Discrete Applied Math. 147(2-3), 245-263 (2005)

7. Gonzalez-Diaz, R., Ion, A., Jimenez, M.J., Poyatos, R.: Incremental-Decremental Algorithm for Computing AT-Models and Persistent Homology. In: Real, P., DiazPernil, D., Molina-Abril, H., Berciano, A., Kropatsch, W. (eds.) CAIP 2011, Part I. LNCS, vol. 6854, pp. 286-293. Springer, Heidelberg (2011)

8. Ghrist, R.: Barcodes: The persistent topology of data. Bulletin of the American Mathematical Society 45, 61-75 (2008)

9. Gutierrez, A., Monaghan, D., Jiménez, M.J., O'Connor, N.E.: Persistent Homology for 3D Reconstruction Evaluation. In: Ferri, M., Frosini, P., Landi, C., Cerri, A., Di Fabio, B. (eds.) CTIC 2012. LNCS, vol. 7309, pp. 139-147. Springer, Heidelberg (2012)

10. Kaibel, V.: Extended formulations in combinatorial optimization. Optima $85,2-7$ (2011)

11. Lamar-León, J., García-Reyes, E.B., Gonzalez-Diaz, R.: Human Gait Identification Using Persistent Homology. In: Alvarez, L., Mejail, M., Gomez, L., Jacobo, J. (eds.) CIARP 2012. LNCS, vol. 7441, pp. 244-251. Springer, Heidelberg (2012)

12. Hatcher, A.: Algebraic Topology. Cambridge University Press (2002)

13. Joswig, M., Pfetsch, M.E.: Computing Optimal Discrete Morse Funcions. Elec. Notes Disc. Math. 17, 191-195 (2004)

14. Kozlov, D.N.: Combinatorial Algebraic Topology. Springer (2008)

15. Lewiner, T., Lopes, H., Tavares, G.: Optimal Discrete Morse Functions for 2manifolds. Comput. Geom. 26, 221-233 (2003)

16. Maver, J., Bajcsy, R.: Occlusions as a guide for planning the next view. IEEE Transactions on Pattern Analysis and Machine Intelligence 15(5), 417-433 (1993)

17. Munkres, J.: Elements of Algebraic Topology. Addison-Wesley Co. (1984)

18. Scott, W.R., Roth, G., Rivest, J.F.: View planning for automated threedimensional object reconstruction and inspection. ACM Computing Surveys 35(1) (2003)

19. Tarabanis, K.A., Allen, P.K., Tsai, R.Y.: A survey of sensor planning in computer vision. IEEE Trans. on Robotics and Automation 11(1), 86-104 (1995)

20. Vanderbeck, F., Wolsey, L.A.: Reformulation and decomposition of integer programs. In: Junger, M., et al. (eds.) 50 Years of Integer Programming 1958-2008, pp. 431-502. Springer (2010)

21. Zomorodian, A., Carlsson, G.: Computing persistent homology. Discrete and Computational Geometry 33(2), 249-274 (2005) 